\title{
Application of Homotopy Analysis Method in One- Dimensional Instability Phenomenon Arising in Inclined Porous Media
}

\author{
Kajal K. Patel", M. N. Mehta, Twinkle R. Singh \\ Department of Applied Mathematics \& Humanities, S. V. National Institute of Technology, Surat, India \\ *Corresponding author: kajal11@gmail.com
}

Received Jaunary 21, 2014; Revised March 06, 2014; Accepted April 24, 2014

\begin{abstract}
During secondary oil recovery process when water is injected in inclined oil formatted area then phenomenon of instability occurs due to viscosity difference of water and oil. The non-linear partial differential equation for this instability phenomenon have been obtained. The Homotopy analysis method has been applied to this governing equation by using appropriate initial and boundary conditions. The guess value of saturation of injected water has been satisfying its initial and boundary conditions. The numerical value and graphical presentation are given by using Maple software and it is concluded that the saturation of injected water is increasing during instability phenomenon in inclined porous media when length of fingers and time increases.
\end{abstract}

Keywords: instability phenomenon, homotopy analysis method, porous matrix, secondary oil recovery process

Cite This Article: Kajal K. Patel, M. N. Mehta, and Twinkle R. Singh, "Application of Homotopy Analysis Method in One-Dimensional Instability Phenomenon Arising in Inclined Porous Media." American Journal of Applied Mathematics and Statistics, vol. 2, no. 3 (2014): 106-114. doi: 10.12691/ajams-2-3-4.

\section{Introduction}

Since last few decades, the study of multiphase flow through porous media has great important in petroleum technology for oil recovery process. In oil recovery process oil can recover through three different stages like primary, secondary and tertiary or enhanced oil recovery. Primary oil recovery is the process in which oil comes out from oil formatted area by natural pressure and it produces only $10 \%$ to $15 \%$ of oil by natural pressure of the reservoir. Remaining oil can recover by injecting water, gas or any chemical. Hence this process is called secondary oil recovery process. In this paper, we consider the phenomenon of instability occurs during water injection in secondary oil recovery process as shown in Figure 1 when water is injected in oil formatted area. Due to the viscosity difference of water and oil, instead of regular displacement of whole front (common interface) the protuberances will occurs with irregular fingers in size and shape. Hence it is called fingering phenomenon. Injection of water is the principal form of the secondary oil recovery because supply of water is often plentiful, inexpensive and it is usually more stable frontal displacement than other form of secondary oil recovery. Due to water injection, oil will displace towards the production well in this way remaining oil can recover in secondary recovery process. In 1972, Bear considered two types of frontal displacement when two or more fluids in motion occupy the porous media. Firstly: Stable displacement and secondly: instable displacement [1]. The stability of water flood depends on the mobility ratio between oil and water, heterogeneity of the porous medium and the interaction of several forces.

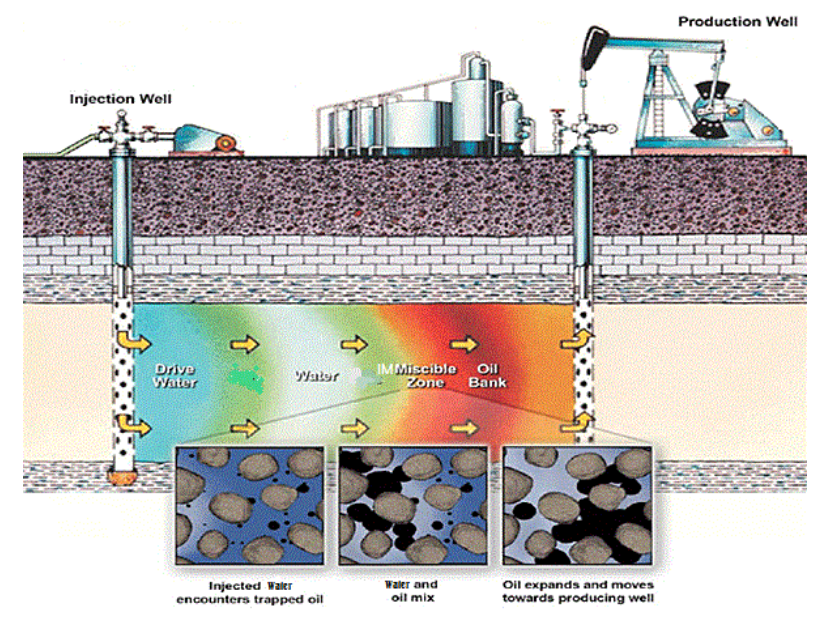

Figure 1. Schematic illustration of water injection in an oil reservoir. Cross section show the distribution of oil and water before and after the water has displaced the oil in porous matrix.

Instabilities may occur in both miscible and immiscible processes, and originate on the interface between two fluids (e.g. oil and water).For displacements according to the Muskat model [12], in which oil and water flow in separate macroscopic regions and gravitational forces are absent. Chuoke et al. [3] determined a condition for instability is that the mobility of the displacing water be higher than that of the displaced oil. He also accounted for capillary effects by defining an effective interfacial 
tension between the fluids in a porous medium analogous to the interfacial tension in the bulk fluids. The first application of the Hele-Shaw cell in viscous fingering problems were made by Saffman and Taylor [17]. As both the velocity of the fluid in a porous medium and the velocity of a fluid in a Hele-Shaw cell obey Darcy's law, Saffman and Taylor suggested that the behavior of a HeleShaw cell could represent flow in a porous medium [17]. This phenomenon is analytically discussed first time by Schidegger and Johnson [19]. They have defined a different approach to this phenomenon in which they have called the statistical of fingers as shown in Figure 2. In the statistical treatment of fingers only the average cross section area occupied by the schematic fingers had been considered, while size and shape of the individual fingers were neglected and the capillary mean pressure of the finger were also ignored. Verma [21] has obtained the statistical behavior of fingering in a heterogeneous porous media with mean capillary pressure. Patel and Mehta [16] have studied this phenomenon by converting the governing equation of fingering phenomenon in the form of Burger's equation and solution obtained in analytical form. Kinjal and Mehta [7] have given a series solution of nonlinear partial differential equation for fingering phenomenon in heterogeneous as well as in homogeneous porous media. Joshi and Mehta (2009) have discussed solution of governing equation by the Group Invariant method in homogenous porous media [6]. Vyas and Mehta have discussed instability phenomenon with magnetic field effect [20]. Meher and Mehta [12] have been obtained an approximate solution of governing equation of instability phenomenon by Adomain Decomposition method. Since governing equation of instability phenomenon is nonlinear partial differential equation of diffusion type, hence it is appropriate to apply Homotopy analysis method to find an approximate solution of the problem.

\section{Statement of the Problem}

For the sake of mathematical study, we consider onedimensional instability phenomenon for which we choose a piece of cylindrical porous matrix from large natural field area and take vertical cross-sectional area of this small finite incline cylindrical porous matrix as a rectangle. Which is incline at small angle $\theta$ with $\mathrm{x}$-axis and its three sides are impermeable expect for one end of cylinder which is designated as common interface at $\mathrm{x}=0$ as shown in Figure 2. Since the water is injected in oil formatted area and water and oil are flowing through porous matrix then it will satisfy Darcy's law [5] for low Reynolds number. Hence cylindrical pieces of incline porous matrix is filled with the oil and water is injected at $\mathrm{x}=0$ hence both will satisfy the equation of continuity.

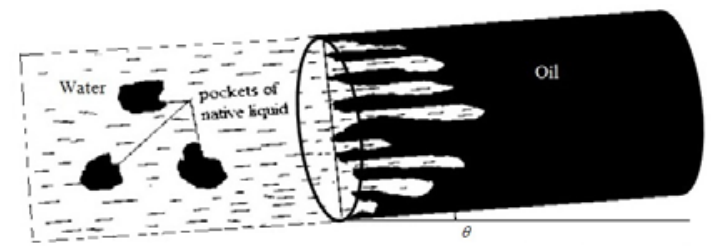

Figure 2. Formation of fingers in the inclined cylindrical piece of porous media

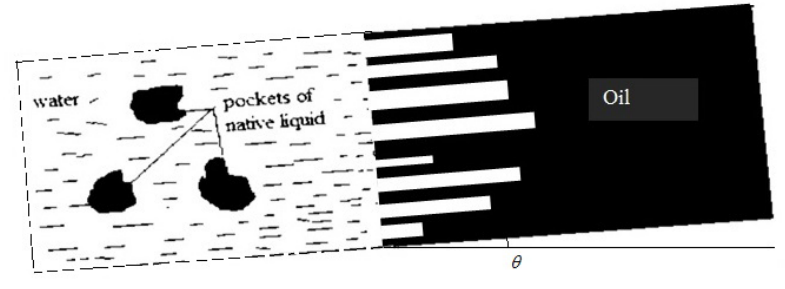

Figure 3. Schematic representation of fingers at level “ $x$ ”

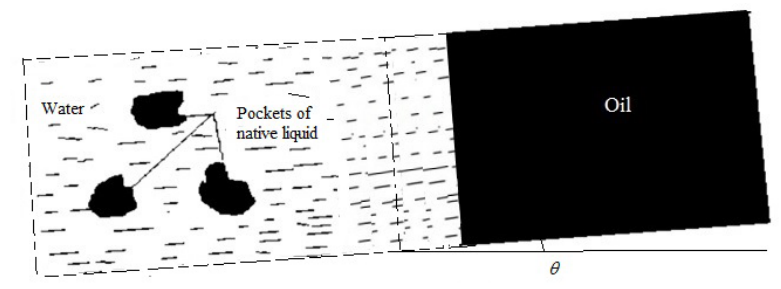

Figure 4. Schematic representation of injected water at level " $x$ "

When water is injected at common interface $\mathrm{x}=0$ then instead of regular displacement of the whole front, small protuberance will occurs due to the water following through interconnected capillaries in the porous matrix due to capillary pressure the irregular fingers occurred with irregular sizes and shapes. To find the area occupy by water in form of saturation of water in different irregular fingers as shown in Figure 2 by white dotted fingers. Schidegger and Johnson [19] suggested schematic presentation of fingers by replacing irregular fingers by rectangular fingers for mathematical study as shown in Figure 3. But still it is difficult to find the saturation occupied in schematic fingers for given length $\mathrm{x}$ and time $t>0$. Hence for one-dimensional study of instability phenomenon by taking average cross-sectional area occupied by schematic finger is considered as saturation of injected water for the length of fingers $x$ for given time $\mathrm{t}>0$ which is rectangular shape as shown by Figure 4 . Here the injection of water is taking place at $x=0$ to native formation of oil formatted (dark area) porous media as shown in Figure 4. We consider here that saturation of water at common interface occupied cross-sectional area is very small saturation $S_{w 0}$. It is also considered for mathematical study that the initial saturation at common interface as average cross-sectional area is very small $S_{w c}$ where $S_{w c}<S_{w 0}$. The comparative study have been carried out for instability phenomenon in homogeneous porous matrix with and without inclination.

This paper presents the instability phenomenon which occurs during the displacement process of two immiscible fluids through homogeneous porous media in secondary oil recovery process.

\section{Mathematical Formulation}

Since water is injected at common interface in inclined homogeneous porous matrix contenting oil which will displaced by injecting water. Hence water and oil both will satisfy Darcy's law given by Bear [1] which gives velocities of water and oil respectively as

$$
V_{i w}=-\frac{k_{i w}}{\mu_{i w}} K\left(\frac{\partial p_{i w}}{\partial x}+\rho_{i w} g \sin \theta\right)
$$




$$
V_{n o}=-\frac{k_{n o}}{\mu_{n o}} K\left(\frac{\partial p_{n o}}{\partial x}+\rho_{n o} g \sin \theta\right)
$$

Since in inclined porous matrix gravitational effect $g$ and angle of inclination play important role in velocities of injected water and native oil so the second terms are added in above equations.

Where $V_{i w}$ and $V_{n o}$ represents velocity of injected water and native oil respectively, $\mathrm{K}$ is the permeability of the homogenous porous medium, $\theta$ is the angle of inclination of porous media measured with the horizontal line in positive direction, $k_{i w}$ and $k_{n o}$ are relative permeability of the injected water and native oil, which are the function of their saturations $S_{i w}$ and $S_{n o}, p_{i w}$ and $p_{n o}$ are pressures of injecting water and displaced native oil, $\rho_{i w}$ and $\rho_{\text {no }}$ are constant densities of injected water and native oil, $\mathrm{g}$ is the acceleration due to gravity, $\mu_{i w}$ and $\mu_{n o}$ are the constant kinematic viscosities of injected water and native oil.

Since water and oil are following in a porous matrix through interconnected capillaries during the phenomenon of instability, due to capillary pressure of water and oil. Injected water and displaced native oil which will satisfy equation of continuity as given by Scheidegger [18] as

$$
\begin{aligned}
& \phi \frac{\partial S_{i w}}{\partial t}+\frac{\partial V_{i w}}{\partial x}=0 \\
& \phi \frac{\partial S_{n o}}{\partial t}+\frac{\partial V_{n o}}{\partial x}=0
\end{aligned}
$$

Where $\phi$ is the porosity of the homogeneous porous medium which is considered here as a constant.

It is also given that the sum of the saturation of the injected water and native oil is equal to unity [18] (i.e. fully saturated) written as

$$
S_{i w}+S_{n o}=1
$$

Further for the instability phenomenon it is necessary to understand the role of capillary pressure. Hence when less viscous water is injected at $x=0$ in oil formatted inclined porous matrix of length $x=L$, the water can flow through interconnected capillaries made by effective pores and it is due to the difference of pressure of native oil and injected water. Which is also a function of saturation of injected water defined by Scheidegger [18] as

$$
p_{c}\left(S_{i w}\right)=p_{n o}-p_{i w}
$$

But Mehta [13] consider that the injected water linearly flowing through small interconnected capillary in homogeneous porous media. Hence he consider capillary pressure $p_{c}$ as linear function of saturation $S_{i w}$ given by

$$
p_{c}=-\beta S_{i w} \text {; is constant }
$$

Here minus sign shows the direction of capillary pressure opposite to the saturation of the injected water. This relation has been considered by many author $[6,7,12,16,20]$ in their research work. Schidegger [18] and Bear [1] had defined this relationship between permeability and saturation of water and oil respectively as

$$
\left.\begin{array}{l}
k_{i w}=S_{i w} \\
k_{n o}=S_{n o}=1-\alpha S_{i w}
\end{array}\right\}
$$

where $\alpha=1.11$ which is constant.

Now substituting the value of seepage velocity of injected water $V_{i w}$ and velocity of native oil $V_{\text {no }}$ from equation (1) and (2) into the continuity equations of (3) and (4) the following coupled partial differential equations as

$$
\begin{gathered}
\phi \frac{\partial S_{i w}}{\partial t}=\frac{\partial}{\partial x}\left[\frac{k_{i w}}{\mu_{i w}} K\left(\frac{\partial p_{i w}}{\partial x}+\rho_{i w} g \sin \theta\right)\right] \\
\phi \frac{\partial S_{n o}}{\partial t}=\frac{\partial}{\partial x}\left[\frac{k_{n o}}{\mu_{n o}} K\left(\frac{\partial p_{n o}}{\partial x}+\rho_{n o} g \sin \theta\right)\right]
\end{gathered}
$$

Eliminating $\frac{\partial p_{i w}}{\partial x}$ from equations (9) by using relation (6) we get

$$
\phi \frac{\partial S_{i w}}{\partial t}=\frac{\partial}{\partial x}\left[\frac{k_{i w}}{\mu_{i w}} K\left\{\left(\frac{\partial p_{n o}}{\partial x}\right)-\left(\frac{\partial p_{c}}{\partial x}\right)+\rho_{i w} g \sin \theta\right\}\right]
$$

Adding equation (10) and (11) and using relation (5) and integrating with respect to $\mathrm{x}$ we get,

$$
\left\{\begin{array}{l}
\left(\frac{k_{i w}}{\mu_{i w}} K+\frac{k_{n o}}{\mu_{n o}} K\right) \frac{\partial p_{n o}}{\partial x}-\left(\frac{k_{i w}}{\mu_{i w}} K\right) \frac{\partial p_{c}}{\partial x} \\
+\left(\frac{k_{i w}}{\mu_{i w}} \rho_{i w} K+\frac{k_{n o}}{\mu_{n o}} \rho_{n o} K\right) g \sin \theta
\end{array}\right\}=-V(t)
$$

Where $\mathrm{V}(\mathrm{t})$ is a constant of integration.

After simplification of (12), we get

$$
\frac{\partial p_{n o}}{\partial x}=\left\{\begin{array}{c}
-\frac{V(t)}{\left(\frac{k_{i w}}{\mu_{i w}} K+\frac{k_{n o}}{\mu_{n o}} K\right)}+\frac{\left(\frac{k_{i w}}{\mu_{i w}} K\right)\left(\frac{\partial p_{c}}{\partial x}\right)}{\left(\frac{k_{i w}}{\mu_{i w}} K+\frac{k_{n o}}{\mu_{n o}} K\right)} \\
-\frac{\left(\frac{k_{i w}}{\mu_{i w}} \rho_{i w} K+\frac{k_{n o}}{\mu_{n o}} \rho_{n o} K\right) g \sin \theta}{\left(\frac{k_{i w}}{\mu_{i w}} K+\frac{k_{n o}}{\mu_{n o}} K\right)}
\end{array}\right\}
$$

Using above value of $\frac{\partial p_{n o}}{\partial x}$ in equation (11) we get

$$
\phi \frac{\partial S_{i w}}{\partial t}=\frac{\partial}{\partial x}\left\{\frac{k_{i w}}{\mu_{i w}} K\left(\begin{array}{c}
-\frac{V(t)}{\left(\frac{k_{i w}}{\mu_{i w}} K+\frac{k_{n o}}{\mu_{n o}} K\right)}-\frac{\left(\frac{k_{n o}}{\mu_{n o}} K\right)\left(\frac{\partial p_{c}}{\partial x}\right)}{\left(\frac{k_{i w}}{\mu_{i w}} K\right)} \\
\left.+\frac{k_{n o}}{\mu_{n o}} K\right) \\
-\frac{\left(\frac{k_{n o}}{\mu_{n o}} K\left(\rho_{n o}-\rho_{i w}\right)\right) g \sin \theta}{\left(\frac{k_{i w}}{\mu_{i w}} K+\frac{k_{n o}}{\mu_{n o}} K\right)}
\end{array}\right)\right\}
$$

Now Oroveanu [15] gave relation of mean pressure by 


$$
p_{n o}=\bar{p}+\frac{1}{2} p_{c}, \bar{p}=\frac{\left(p_{n o}+p_{i w}\right)}{2}
$$

where $\bar{p}$ is the constant mean pressure.

$$
\frac{\partial p_{n o}}{\partial x}=\frac{1}{2} \frac{\partial p_{c}}{\partial x}
$$

To determine the value of $V(t)$ we use relation (16) in equation (13) and after simplification we get,

$$
-V(t)=\left\{\begin{array}{l}
\frac{1}{2}\left(\frac{k_{n o}}{\mu_{n o}} K-\frac{k_{i w}}{\mu_{i w}} K\right) \frac{\partial p_{c}}{\partial x} \\
+\left(\frac{k_{n o}}{\mu_{n o}} \rho_{n o} K+\frac{k_{i w}}{\mu_{i w}} \rho_{i w} K\right) g \sin \theta
\end{array}\right\}
$$

Hence using above value of $\mathrm{V}(\mathrm{t})$ in (14) and after simplification we get,

$$
\phi \frac{\partial S_{i w}}{\partial t}=\frac{\partial}{\partial x}\left\{\frac{k_{i w}}{\mu_{i w}} K\left(-\frac{1}{2} \frac{\partial p_{c}}{\partial x}\right)+\frac{k_{i w}}{\mu_{i w}} \rho_{i w} K g \sin \theta\right\}
$$

For more simplification of above equation of motion we use relation of $p_{c}$ and $k_{\text {in }}$ from equation (6) and (8) respectively in equation (18) we get,

$$
\phi \frac{\partial S_{i w}}{\partial t}=\frac{\beta K}{2 \mu_{i w}} \frac{\partial}{\partial x}\left(S_{i w} \frac{\partial S_{i w}}{\partial x}\right)+\frac{K \rho_{i w} g}{\mu_{i w}} \sin \theta \frac{\partial S_{i w}}{\partial x}
$$

Which is non-linear partial differential equation of motion for the saturation of injected water during instability phenomenon occurring in secondary oil recovery process in incline homogeneous porous matrix.

To solve nonlinear equation (19), it is necessary to choose appropriate boundary and initial condition at common interface $x=0$. As par given statement, we consider here that when water is injected at common interface $\mathrm{x}=0$ the saturation of injected water is $\mathrm{S}_{\mathrm{w} 0}$ which is very small for given time $t>0$.

$$
\text { i.e. } S_{i w}(0, t)=S_{w 0} \text { at } \mathrm{x}=0 \text { for } \mathrm{t}>0
$$

It is also considered in statement that the initial saturation of injected water is $S_{w c}$.

i.e. $S_{i w}(x, 0)=S_{w c}=S_{w 0} e^{\frac{x}{L}}$ at $\mathrm{t}=0$ for $\mathrm{x}>0, \mathrm{~L}$ is length of porous matrix where

$$
0 \leq S_{w c}<S_{w 0}
$$

\section{The Solution with HAM}

To find the solution of non-linear partial differential equation (19) for phenomenon of instability together with the condition (20)-(21). Choosing dimensionless variable

$$
X=\frac{x}{L} \text { and } T=\frac{K}{\phi L^{2} \mu_{i w}} t
$$

The equation (19) together with condition (20-21) will be converted into dimensionless form as

$$
\frac{\partial S_{i w}}{\partial T}=\varepsilon S_{i w} \frac{\partial^{2} S_{i w}}{\partial X^{2}}+\varepsilon\left(\frac{\partial S_{i w}}{\partial X}\right)^{2}+A \frac{\partial S_{i w}}{\partial X}
$$

where $\varepsilon=\frac{\beta}{2}$, and $A=\rho_{i w} g \sin \theta L$ units which is constant.

$$
\begin{gathered}
S_{i w}(0, T)=S_{w 0} \text { at } \mathrm{X}=0 \text { for } \mathrm{T}>0 \\
S_{i w}(X, 0)=S_{w 0} e^{X} \text { at } \mathrm{T}=0 \text { for } \mathrm{X}>0,0 \leq S_{w c}<S_{w 0}
\end{gathered}
$$

To solve the equation (22) together with condition (23) and (24) we use Homotopy analysis method. For this method first necessary step is to guess value of saturation $S_{i w}$ which satisfies above conditions (23) and (24) as

$$
S^{*}(X, T ; q)=\left(S_{w 0} e^{X}+\frac{T}{2} X\right)+q^{m}
$$

Let the saturation of injected water at common interface $\mathrm{X}=0$ for embedded parameter $\mathrm{q}=0$ is $S_{w 0}$ then

$$
S^{*}(0, T ; 0)=S_{w 0}
$$

Let us consider non-linear partial differential equation (22) can be expressed as,

$$
\mathbb{N}\left[S^{*}(\mathrm{X}, T ; \mathrm{q})\right]=0
$$

Where $\mathbb{N}$ is a nonlinear operator, $S^{*}(\mathrm{X}, T ; \mathrm{q})$ is considered as unknown function which represent saturation of injected water for any distance $X$ for given time $T \geq 0$ for $0 \leq q \leq 1$. We use auxiliary linear operator $\mathfrak{I}=\partial / \partial T$ and initial first approximation of the saturation of injected water for $q=0$ will be $S_{i 0}(\mathrm{X}, T)=\left(S_{w 0} e^{X}+\frac{T}{2} X\right) \quad$ to construct the corresponding zero ${ }^{\text {th }}$-order deformation equation. As the auxiliary linear operator which satisfies $\mathfrak{I}\left[C_{1}\right]=0$, where $\mathrm{C}_{1}$ is arbitrary constant. We construct a homotopy as [11],

$$
\begin{aligned}
& \mathrm{H}\left[S^{*}(\mathrm{X}, T ; \mathrm{q}) ; \mathrm{S}_{i 0}(\mathrm{X}, T), H(\mathrm{X}, T), \hbar, \mathrm{q}\right] \\
& =(1-q)\left\{\begin{array}{l}
\mathfrak{I}\left[S^{*}(\mathrm{X}, T ; \mathrm{q})-S_{i 0}(\mathrm{X}, T)\right] \\
-q \hbar H(\mathrm{X}, T) \mathbb{N}\left[S^{*}(\mathrm{X}, T ; \mathrm{q})\right]
\end{array}\right\}
\end{aligned}
$$

Enforcing the Homotopy (28) to be zero [11],

$$
\mathrm{H}\left[S^{*}(\mathrm{X}, T ; \mathrm{q}) ; \mathrm{S}_{i 0}(\mathrm{X}, T), H(\mathrm{X}, T), \hbar, \mathrm{q}\right]=0
$$

Establish the zero-order deformation equation of instability phenomenon as [11],

$$
\begin{aligned}
& (1-q) \Im\left[S^{*}(\mathrm{X}, T ; \mathrm{q})-S_{i 0}(\mathrm{X}, T)\right] \\
& =q \hbar H(\mathrm{X}, T) \mathbb{N}\left[S^{*}(\mathrm{X}, T ; \mathrm{q})\right]
\end{aligned}
$$

Where $S_{i 0}(\mathrm{X}, T)$ denote initial first approximation of the saturation of injected water of the exact solution of the saturation of injected water $\mathrm{S}_{i w}(\mathrm{X}, T)$ which we want to find. For which $\hbar \neq 0$ is an auxiliary parameter, $H(\mathrm{X}, T) \neq 0$ is an auxiliary function [11], $q \in[0,1]$ is an embedding parameter and $\mathfrak{I}$ is an auxiliary linear operator with the property 


$$
\mathfrak{I}\left[S^{*}(\mathrm{X}, T ; \mathrm{q})\right]=0 \text { when } S^{*}(\mathrm{X}, T ; \mathrm{q})=0
$$

Since auxiliary parameter $\hbar$ and auxiliary function $H(X, T)$ is nonzero.

For $q=0$ and $q=1$ we will get respectively,

$$
S^{*}(\mathrm{X}, T ; 0)=S_{i 0}(\mathrm{X}, T) \text { and } S^{*}(\mathrm{X}, T ; 1)=S_{i w}(\mathrm{X}, T)(30)
$$

According to (28) as the embedding parameter $q$ increases from 0 to 1 , solution $S^{*}(\mathrm{X}, T ; \mathrm{q})$ varies continuously from the initial guess $S_{i 0}(\mathrm{X}, T)$ of the saturation of the injected water to the required solution $S_{i w}(\mathrm{X}, T)$ (solution gradually improved from $\mathrm{X}=0$ to the end of rectangle as shown in Figure 4). As [10] considered saturation of injected water and its solution is assumed as

$$
S^{*}(\mathrm{X}, T ; \varepsilon)=S^{*}(\mathrm{X}, T ; 0)+\sum_{m=1}^{\infty} S_{i m}(\mathrm{X}, T) \mathrm{q}^{m}
$$

Where

$$
S_{i m}(\mathrm{X}, T)=\left.\frac{1}{m !} \frac{\partial^{m} S^{*}(\mathrm{X}, T ; \mathrm{q})}{\partial q^{m}}\right|_{q=0}
$$

i.e. the saturation of injected water is a function of distance $\mathrm{X}$, and time $\mathrm{T}$ for any parametric value $q$ is expressed as, the saturation of injected fluid at common interface $S^{*}(\mathrm{X}, T ; 0)$ and sum of schematic presentation of fingers for saturation of injected fluid as given by Scheidegger for different value of parameter $q$ is expressed as saturation of injected fluid at time $T=0$ is $S_{i 0}(\mathrm{X}, T)$ and sum of saturation of injected fluid fingers are $S_{i 1}(\mathrm{X}, T), S_{i 2}(\mathrm{X}, T), \ldots$ at different time $\mathrm{T}$ for different value of parameter $q$. Here, the series (35) is called homotopy-series; the series (31) is called homotopy series solution of $\mathbb{N}\left[S^{*}(\mathrm{X}, T ; \mathrm{q})\right]=0$ and $S_{i m}(\mathrm{X}, T)$ is called the $\mathrm{m}^{\text {th }}$-order derivative of $S^{*}$. Auxiliary parameter $\hbar$ in homotopy-series (31) can be regard as iteration factor and is widely used in numerical computations. It is well known that the properly chosen iteration factor can ensure the convergence of homotopy series (31) is depending upon the value of $\hbar$, one can ensure that convergent of homotopy series, solution simply by means of choosing the proper value of $\hbar$ as shown by Liao $[8,9,10,11]$. If the auxiliary linear operator, the initial guess, the auxiliary parameter $\hbar$, the auxiliary function $H(X, T)$ are so properly chosen, the series (31) converges at $q=1$.

Hence the saturation of injected fluid can be expressed as,

$$
S_{i w}(\mathrm{X}, T)=S_{i 0}(\mathrm{X}, T)+\sum_{m=1}^{\infty} S_{i m}(\mathrm{X}, T)
$$

and $S_{i m}(\mathrm{X}, T)$ will be calculated by equation (38).

This must be one of the solution of original non-linear partial differential equation (22) for saturation of injected fluid. According to the definition (32), the governing equation can be deduced from the zero-order deformation equation (26). Define the vector

$$
\vec{S}_{\text {in }}=\left\{S_{i 0}(\mathrm{X}, T), \mathrm{S}_{i 1}(\mathrm{X}, T), \ldots \mathrm{S}_{\text {in }}(\mathrm{X}, T)\right\}
$$

Differentiating equation (30) $\mathrm{m}$ times with respect to the embedding parameter $q$ and then setting $q=1$ and finally dividing them by $m$ !, we have the so-called $\mathrm{m}^{\text {th }}$ order deformation equation for saturation of injected fluid water $\mathrm{S}$ will be as [11]

$$
\begin{aligned}
\mathfrak{I}\left[S_{m}(\mathrm{X}, T)-\chi_{m} S_{m-1}(\mathrm{X}, T)\right] \\
=q \hbar H(\mathrm{X}, T) R_{m}\left(\vec{S}_{m-1}, \mathrm{X}, T\right)
\end{aligned}
$$

Where

$R_{m}\left(\vec{S}_{i(m-1)}, \mathrm{X}, T\right)=\left.\frac{1}{(m-1) !} \frac{\partial^{m-1} \mathbb{N}\left[S^{*}(\mathrm{X}, T ; \mathrm{q})\right]}{\partial q^{m-1}}\right|_{q=0}$

And,

$$
\chi_{m}= \begin{cases}0, & m \leq 1 \\ 1, & m>1\end{cases}
$$

It should be emphasized that $S_{i m}(\mathrm{X}, T)$ for $m \geq 1$ is governed by the linear equation (32) with the linear boundary condition that come from the original problem, which can solve by symbolic computation software Maple as bellow. The rule of solution expression as given by equation (22) and equation (26), the auxiliary function independent of $q$ can be chosen as $H(X, T)=1$ [11].

According to (30) and taking inverse of equation (34) we get,

$$
\begin{gathered}
S_{i m}(\mathrm{X}, T)=\chi_{m} S_{i(m-1)}(\mathrm{X}, T) \\
+\hbar \mathfrak{I}^{-1}\left[R_{m}\left(\vec{S}_{i(m-1)}, \mathrm{X}, T\right)\right] \\
R_{m}\left(\vec{S}_{i(m-1)}, \mathrm{X}, T\right)=\frac{1}{m !} \frac{\partial^{m-1} \mathbb{N}\left[S^{*}(\mathrm{X}, T ; \mathrm{q})\right]}{\partial q^{m-1}}
\end{gathered}
$$

In this way, we get $S_{i m}(\mathrm{X}, T)$ for $m=1,2,3 \ldots$ successively by using Maple software as,

$$
S_{i 1}(\mathrm{X}, T)=-\frac{1}{12} \hbar T\left(\begin{array}{l}
\varepsilon T^{2}+3 \varepsilon S_{w 0} T X e^{X}+6 \varepsilon T S_{w 0} e^{X} \\
+3 A T-6 X+24 \varepsilon S_{w 0}^{2} e^{2 X} \\
+12 A S_{w 0} e^{X}
\end{array}\right)
$$

$S_{i 2}(\mathrm{X}, T)=$

$$
\frac{1}{96} \hbar T\left(\begin{array}{l}
-192 A S_{w 0} e^{X}-48 \varepsilon T S_{w 0} e^{X}-24 \varepsilon T X S_{w 0} e^{X} \\
-192 \varepsilon S_{w 0}^{2} e^{2 X}+48 X-8 \varepsilon T^{2}-48 A T \\
+24 \varepsilon \hbar A X T^{2} S_{w 0} e^{X}-24 \hbar \varepsilon T^{2}-48 \hbar A T \\
+3 \hbar \varepsilon^{2} T^{3} X^{2} S_{w 0} e^{X}+18 \hbar \varepsilon^{2} X T^{3} e^{X} S_{w 0} \\
+64 \hbar \varepsilon A T^{2} S_{w 0} e^{X}-48 \hbar \varepsilon T X S_{w 0} e^{X} \\
+20 \hbar \varepsilon^{2} T^{3} S_{w 0} e^{X}-96 \hbar \varepsilon T S_{w 0} e^{X} \\
+48 \hbar A^{2} T S_{w 0} e^{X}-96 \hbar A S_{w 0} e^{X} \\
+224 \hbar \varepsilon^{2} T^{2} S_{w 0}^{2} e^{2 X}+864 \hbar \varepsilon^{2} T S_{w 0}^{3} e^{3 X} \\
-192 \hbar \varepsilon S_{w 0}^{2} e^{2 X}+160 \hbar \varepsilon^{2} X T^{2} S_{w 0}^{2} e^{2 X} \\
+384 \hbar \varepsilon A T S_{w 0}^{2} e^{2 X}+48 \hbar X
\end{array}\right)
$$


Using initial guess value for the saturation of injected water from equation (22) and successive saturation of injected water into the native oil form (39) and (32) etc. and using in equation (34), we get

$$
\begin{aligned}
& \mathrm{S}_{i w}(\mathrm{X}, T)= \\
& \left(\begin{array}{l}
\left(S_{w 0} e^{X}+\frac{T}{4} X\right)-\frac{1}{12} \hbar T\left(\begin{array}{l}
\varepsilon T^{2}+3 \varepsilon S_{w 0} T X e^{X} \\
+6 \varepsilon S_{w 0} e^{X} T+3 A T-6 X \\
+24 \varepsilon S_{w 0}^{2} e^{2 X}+12 A S_{w 0} e^{X}
\end{array}\right) \\
+\frac{1}{96} \hbar T\left(\begin{array}{l}
-192 A S_{w 0} e^{X}-48 \varepsilon T S_{w 0} e^{X} \\
-24 \varepsilon T X S_{w 0} e^{X}-192 \varepsilon S_{w 0}^{2} e^{2 X} \\
+48 X-8 \varepsilon T^{2}-48 A T+24 \varepsilon \hbar A X T^{2} S_{w 0} e^{X} \\
-24 \hbar \varepsilon T^{2}+384 \hbar \varepsilon A T S_{w 0}^{2} e^{2 X} \\
+48 \hbar X+3 \hbar \varepsilon^{2} T^{3} X^{2} S_{w 0} e^{X} \\
+18 \hbar \varepsilon^{2} X T^{3} e^{X} S_{w 0}+64 \hbar \varepsilon A T^{2} S_{w 0} e^{X} \\
-48 \hbar \varepsilon T X S_{w 0} e^{X}+20 \hbar \varepsilon^{2} T^{3} S_{w 0} e^{X} \\
-96 \hbar \varepsilon T S_{w 0} e^{X}+48 \hbar A^{2} T S_{w 0} e^{X} \\
-96 \hbar A S_{w 0} e^{X}+224 \hbar \varepsilon^{2} T^{2} S_{w 0}^{2} e^{2 X} \\
+864 \hbar \varepsilon^{2} T S_{w 0}^{3} e^{3 X}-192 \hbar \varepsilon S_{w 0}^{2} e^{2 X} \\
+160 \hbar \varepsilon^{2} X T^{2} S_{w 0}^{2} e^{2 X}-48 \hbar A T
\end{array}\right.
\end{array}\right\}+
\end{aligned}
$$

Where $S_{i 1}(\mathrm{X}, T), \mathrm{S}_{i 2}(\mathrm{X}, T), \ldots$ are given by equation (28) and (29) respectively represents saturation of the injected water into the native oil at any time $\mathrm{T}$ for the horizontal distance $X$ for $T>0$. The solution is an infinite series solution, which represents the approximate value of saturation of injected water for time $T>0$. Which is convergent at $q=1$ for auxiliary parameter $\hbar=0.02$.

Thus the saturation of injected water into the native oil is expressed in terms of exponentials of function of $\mathrm{X}$ and time $T>0$, which depends on first guess value of the solution (25) only. Equation (41) represents the saturation of injected water in oil formatted region $X \geq 0$ of instability phenomenon in homogeneous porous media with small inclination when injected water is injected with external injecting force at common interface during secondary oil recovery process.

\section{Numerical and Graphical Presentation with Inclination}

Maple coding has been used to obtain numerical and graphical presentations of equation (41). Figure 5 represents the graph of $S_{i w}(X, T)$ vs. distance $\mathrm{X}$ for time $T=0.1,0.2,0.3,0.4,0.5,0.6,0.7,0.8,0.9,1.0$ and fixed value $\quad S_{w 0}=0.1 \quad, \quad \varepsilon=0.05 \quad$ and $A=\rho_{i w} g L \sin \theta=0.1 \times 9.8 \times 2 \times \sin 15=0.5176$. Table 1

\begin{tabular}{|c|c|c|c|c|c|c|c|c|c|c|}
\hline \multirow{2}{*}{$\begin{array}{c}\text { Distance } \\
\mathrm{X}\end{array}$} & \multicolumn{10}{|c|}{ Saturation of the injected water in inclined porous matrix } \\
\hline & $\mathrm{T}=0.1$ & $\mathrm{~T}=0.2$ & $\mathrm{~T}=0.3$ & $\mathrm{~T}=0.4$ & $\mathrm{~T}=0.5$ & $\mathrm{~T}=0.6$ & $\mathrm{~T}=0.7$ & $\mathrm{~T}=0.8$ & $\mathrm{~T}=0.9$ & $\mathrm{~T}=1.0$ \\
\hline 0.1 & 0.1153 & 0.1198 & 0.1245 & 0.1288 & 0.1329 & 0.1371 & 0.1409 & 0.1447 & 0.1483 & 0.1517 \\
\hline 0.2 & 0.1321 & 0.1419 & 0.1515 & 0.1609 & 0.1703 & 0.1795 & 0.1885 & 0.1974 & 0.2061 & 0.2147 \\
\hline 0.3 & 0.1501 & 0.1649 & 0.1798 & 0.1944 & 0.2809 & 0.2232 & 0.2373 & 0.2513 & 0.2652 & 0.2789 \\
\hline 0.4 & 0.1694 & 0.1895 & 0.2094 & 0.2291 & 0.2487 & 0.2681 & 0.2874 & 0.3066 & 0.3256 & 0.3444 \\
\hline 0.5 & 0.1902 & 0.2154 & 0.2404 & 0.2653 & 0.2889 & 03146 & 0.3389 & 0.3633 & 0.3874 & 0.4113 \\
\hline 0.6 & 0.2127 & 0.243 & 0.2731 & 0.3031 & 0.3329 & 0.3627 & 0.3922 & 0.4216 & 0.4508 & 0.4799 \\
\hline 0.7 & 0.2369 & 0.2724 & 0.3076 & 0.3428 & 0.3777 & 0.4125 & 0.4472 & 0.4817 & 0.5159 & 0.5502 \\
\hline 0.8 & 0.2633 & 0.3038 & 0.3442 & 0.3844 & 0.4244 & 0.4643 & 0.5041 & 0.5437 & 0.5832 & 0.6224 \\
\hline 0.9 & 0.2918 & 0.3374 & 0.3829 & 0.4282 & 0.4733 & 0.5184 & 0.5632 & 0.6079 & 0.6525 & 0.6968 \\
\hline 1.0 & 0.3227 & 0.3735 & 0.424 & 0.4744 & 0.5247 & 0.5748 & 0.6247 & 0.6745 & 0.7242 & 0.7739 \\
\hline
\end{tabular}
indicates the numerical value for Figure 5. All tabular values used for the graphical representations of the instability phenomenon, which shows the behavior of the saturation of injected water. The convergence of the Homotopy series (41) is dependent upon the value of convergence-parameter $\hbar[8,9,10,11]$. Therefore we choose proper value of the convergence-parameter $\hbar=0.02$ to obtain convergent Homotopy-series solution [10].

Table 1. Numerical Values of the Saturation of Injected Water in Homogeneous Porous Matrix with Small Inclination $\theta=15^{\circ}$

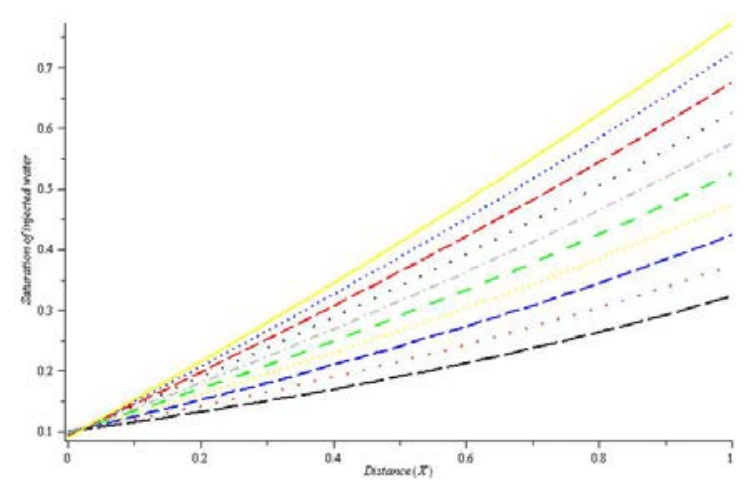

Figure 5. Saturation of injected water at different distance $X$ for fixed time $\mathrm{T}=0.1,0.2,0.3,0.4,0.5,0.6,0.7,0.8,0.9,1.0$ in homogeneous porous matrix with small inclination $\theta=15^{0}$ where $\varepsilon=0.05, S_{w 0}=0.1, \hbar=0.02, \mathrm{~A}=0.5176$

\section{Deduction of instability phenomenon in homogeneous porous media without inclination}

For discussing this case, we put $\theta=0^{\circ}$ in equation 1 and 2 so that the equation (22) becomes

$$
\frac{\partial S_{i w}}{\partial T}=\varepsilon S_{i w} \frac{\partial^{2} S_{i w}}{\partial X^{2}}+\varepsilon\left(\frac{\partial S_{i w}}{\partial X}\right)^{2}
$$

As consider the same condition (23) and (24) and same guess value (25).

Above equation (42) is nonlinear partial differential equation of motion for the saturation of injected water during instability phenomenon occurring in secondary oil 
recovery process in homogeneous porous matrix. The solution of equation (42) can be obtained as [10].

$$
\begin{aligned}
& S_{i w}(X, T)= \\
& \left(\begin{array}{l}
\left.S_{w 0} e^{X}+\frac{T}{2} X\right)-\frac{1}{12} \hbar T\left(\begin{array}{l}
3 \varepsilon T X S_{w 0} e^{X} \\
+6 \varepsilon T S_{w 0} e^{X} \\
-6 X+24 \varepsilon S_{w 0}^{2} e^{2 X} \\
+\varepsilon T^{2}
\end{array}\right) \\
+\frac{1}{96} \hbar T\left(\begin{array}{l}
3 \hbar \varepsilon^{2} S_{w 0} X^{2} T^{3} e^{X}+18 \hbar \varepsilon^{2} S_{w 0} X T^{3} e^{X} \\
+20 \hbar \varepsilon^{2} S_{w 0} T^{3} e^{X}+160 \hbar \varepsilon^{2} S_{w 0}^{2} X T^{2} e^{2 X} \\
+224 \hbar \varepsilon^{2} S_{w 0}^{2} T^{2} e^{2 X}-24 \hbar \varepsilon T^{2} \\
+864 \hbar \varepsilon^{2} S_{w 0}^{3} T e^{3 X}-8 \varepsilon T^{2} \\
-96 \hbar \varepsilon S_{w 0} T e^{X}+48 \hbar X \\
-192 \hbar \varepsilon S_{w 0}^{2} e^{2 X}+48 X \\
-24 \varepsilon S_{w 0} X T e^{X}-48 \varepsilon S_{w 0} T e^{X} \\
-192 \varepsilon S_{w 0}^{2} e^{2 X}-48 \hbar \varepsilon S_{w 0} X T e^{X}
\end{array}\right)+\ldots
\end{array}\right\}
\end{aligned}
$$

Equation (43) represents the saturation of injected water during instability phenomenon in homogeneous porous matrix without inclination.

\section{Numerical and Graphical Presentation without inclination}

Numerical and graphical presentations of equation (43). Figure 6 represents the graph of $S_{i w}(X, T)$ vs. distance $\mathrm{X}$ for time $T=0.1,0.2,0.3,0.4,0.5,0.6,0.7,0.8,0.9,1.0$ and fixed value $S_{w 0}=0.1$, and $\varepsilon=0.05$ Table II indicates the numerical value for Figure 6. The convergence of the Homotopy series (41) is dependent upon the value of convergence-parameter $\hbar \quad[8,9,10,11]$. Therefore we choose proper value of the convergence-parameter $\hbar=0.02$ to obtain convergent Homotopy-series solution [10].

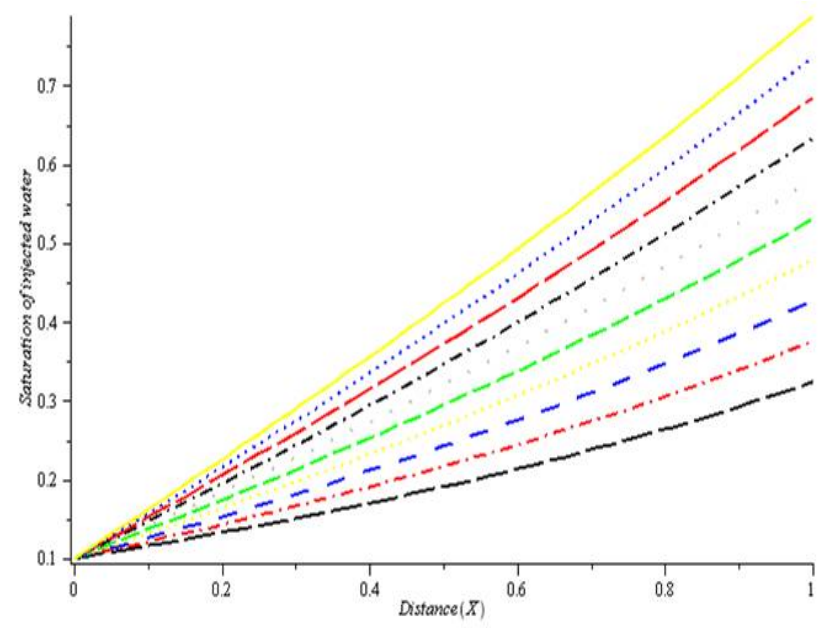

Figure 6. Saturation of injected water at different distance $X$ for fixed time $\mathrm{T}=0.1,0.2,0.3,0.4,0.5,0.6,0.7,0.8,0.9,1.0$ in homogeneous

\begin{tabular}{|c|c|c|c|c|c|c|c|c|c|c|}
\hline \multirow{2}{*}{$\begin{array}{c}\text { Distance } \\
\mathrm{X}\end{array}$} & \multicolumn{10}{|c|}{ Saturation of the injected water in inclined porous matrix } \\
\hline & $\mathrm{T}=0.1$ & $\mathrm{~T}=0.2$ & $\mathrm{~T}=0.3$ & $\mathrm{~T}=0.4$ & $\mathrm{~T}=0.5$ & $\mathrm{~T}=0.6$ & $\mathrm{~T}=0.7$ & $\mathrm{~T}=0.8$ & $\mathrm{~T}=0.9$ & $\mathrm{~T}=1.0$ \\
\hline 0.1 & 0.1157 & 0.1209 & 0.1261 & 0.1312 & 0.1364 & 0.1415 & 0.1467 & 0.1518 & 0.1569 & 0.1619 \\
\hline 0.2 & 0.1325 & 0.1429 & 0.1532 & 0.1635 & 0.1739 & 0.1842 & 0.1945 & 0.2048 & 0.2151 & 0.2253 \\
\hline 0.3 & 0.1505 & 0.1661 & 0.1816 & 0.1971 & 0.2126 & 0.2279 & 0.2435 & 0.2589 & 0.2745 & 0.2899 \\
\hline 0.4 & 0.1699 & 0.1906 & 0.2113 & 0.2319 & 0.2526 & 0.2733 & 0.2939 & 0.3146 & 0.3352 & 0.3558 \\
\hline 0.5 & 0.1908 & 0.2166 & 0.2425 & 0.2683 & 0.2942 & 0.3189 & 0.3456 & 0.3716 & 0.3974 & 0.4232 \\
\hline 0.6 & 0.2133 & 0.2443 & 0.2753 & 0.3063 & 0.3373 & 0.3683 & 0.3993 & 0.4303 & 0.4613 & 0.4922 \\
\hline 0.7 & 0.2376 & 0.2738 & 0.3059 & 0.3462 & 0.3824 & 0.4185 & 0.4547 & 0.4908 & 0.5269 & 0.5631 \\
\hline 0.8 & 0.2639 & 0.3053 & 0.3467 & 0.3879 & 0.4294 & 0.4707 & 0.5119 & 0.5533 & 0.5946 & 0.6359 \\
\hline 0.9 & 0.2926 & 0.3391 & 0.3856 & 0.4321 & 0.4786 & 0.5251 & 0.5716 & 0.6181 & 0.6645 & 0.7109 \\
\hline 1.0 & 0.3236 & 0.3753 & 0.4269 & 0.4787 & 0.5303 & 0.5819 & 0.6336 & 0.6853 & 0.7369 & 0.7885 \\
\hline
\end{tabular}
porous matrix without inclination and $\varepsilon=0.05, S_{w 0}=0.1, \hbar=0.02, \mathrm{~A}=0.5176$

The saturation of injected water in horizontal

8. Comparative Study of Saturation of Injected Water in Instability Phenomenon with and without Inclined Homogeneous Porous Matrix homogeneous porous matrix will be faster than saturation of injected water in inclined homogeneous porous matrix due to absents of gravitational force and angle of inclination. The comparative study of instability phenomenon also shows by the Table 3 that numerical values of saturation of injected water in horizontal homogeneous porous matrix is more than numerical value in inclined homogeneous porous matrix. 
Table 3. Comparative Numerical Value of Saturation of Injected Water With and Without Inclined Porous Matrix

\begin{tabular}{|c|c|c|c|c|c|c|c|c|c|c|}
\hline Distance & \multicolumn{10}{|c|}{ Saturation of the injected water in inclined porous matrix } \\
\hline \multirow[b]{2}{*}{$\mathrm{X}$} & \multicolumn{2}{|c|}{$\mathrm{T}=0.1$} & \multicolumn{2}{|c|}{$\mathrm{T}=0.2$} & \multicolumn{2}{|c|}{$\mathrm{T}=0.3$} & \multicolumn{2}{|c|}{$\mathrm{T}=0.4$} & \multicolumn{2}{|c|}{$\mathrm{T}=0.5$} \\
\hline & $\begin{array}{c}\text { with } \\
\text { incline }\end{array}$ & $\begin{array}{l}\text { without } \\
\text { incline }\end{array}$ & $\begin{array}{c}\text { with } \\
\text { incline }\end{array}$ & $\begin{array}{l}\text { without } \\
\text { incline }\end{array}$ & $\begin{array}{c}\text { with } \\
\text { incline }\end{array}$ & $\begin{array}{l}\text { without } \\
\text { incline }\end{array}$ & $\begin{array}{c}\text { with } \\
\text { incline }\end{array}$ & $\begin{array}{l}\text { without } \\
\text { incline }\end{array}$ & $\begin{array}{c}\text { with } \\
\text { incline }\end{array}$ & $\begin{array}{l}\text { without } \\
\text { incline }\end{array}$ \\
\hline 0.1 & 0.1153 & 0.1157 & 0.1189 & 0.1209 & 0.1245 & 0.1261 & 0.1288 & 0.1312 & 0.1329 & 0.1364 \\
\hline 0.2 & 0.1321 & 0.132 & 0.1719 & 0.1429 & 0.1515 & 0.1532 & 0.161 & 0.1635 & 0.1703 & 0.1739 \\
\hline 0.3 & 0.1501 & 0.1505 & 0.1649 & 0.1661 & 0.1798 & 0.1816 & 0.1944 & 0.1971 & 0.2089 & 0.2126 \\
\hline 0.4 & 0.1694 & 0.1699 & 0.1895 & 0.1906 & 0.2094 & 0.2113 & 0.2291 & 0.2319 & 0.2487 & 0.2526 \\
\hline 0.5 & 0.1902 & 0.1908 & 0.2454 & 0.2166 & 0.2404 & 0.2425 & 0.2653 & 0.2683 & 0.2889 & 0.2942 \\
\hline 0.6 & 0.2127 & 0.2133 & 0.2429 & 0.2443 & 0.2731 & 0.2753 & 0.3031 & 0.3063 & 0.3329 & 0.3373 \\
\hline 0.7 & 0.2369 & 0.2376 & 0.2724 & 0.2738 & 0.3076 & 0.3089 & 0.3428 & 0.3462 & 0.3777 & 0.3824 \\
\hline 0.8 & 0.2633 & 0.2639 & 0.3038 & 0.3053 & 0.3442 & 0.3467 & 0.3844 & 0.388 & 0.4244 & 0.4294 \\
\hline 0.9 & 0.2918 & 0.2926 & 0.3374 & 0.3391 & 0.3829 & 0.3856 & 0.4282 & 0.4321 & 0.4733 & 0.4786 \\
\hline 1.0 & 0.3227 & 0.3236 & 0.3735 & 0.3753 & 0.424 & 0.427 & 0.4744 & 0.4787 & 0.5247 & 0.5303 \\
\hline \multirow[b]{2}{*}{$\mathrm{X}$} & \multicolumn{2}{|c|}{$\mathrm{T}=0.6$} & \multicolumn{2}{|c|}{$\mathrm{T}=0.7$} & \multicolumn{2}{|c|}{$\mathrm{T}=0.8$} & \multicolumn{2}{|c|}{$\mathrm{T}=0.9$} & \multicolumn{2}{|c|}{$\mathrm{T}=1.0$} \\
\hline & $\begin{array}{c}\text { with } \\
\text { incline }\end{array}$ & $\begin{array}{l}\text { without } \\
\text { incline }\end{array}$ & $\begin{array}{c}\text { with } \\
\text { incline }\end{array}$ & $\begin{array}{l}\text { without } \\
\text { incline }\end{array}$ & $\begin{array}{l}\text { with } \\
\text { incline }\end{array}$ & $\begin{array}{l}\text { without } \\
\text { incline }\end{array}$ & $\begin{array}{c}\text { with } \\
\text { incline }\end{array}$ & $\begin{array}{l}\text { without } \\
\text { incline }\end{array}$ & $\begin{array}{c}\text { with } \\
\text { incline }\end{array}$ & $\begin{array}{l}\text { without } \\
\text { incline }\end{array}$ \\
\hline 0.1 & 0.1371 & 0.1415 & 0.1409 & 0.1467 & 0.1447 & 0.1518 & 0.1483 & 0.1569 & 0.1517 & 0.1619 \\
\hline 0.2 & 0.1795 & 0.1842 & 0.1885 & 0.1945 & 0.1974 & 0.1048 & 0.2061 & 0.2151 & 0.2147 & 0.2253 \\
\hline 0.3 & 0.2232 & 0.228 & 0.2373 & 0.2435 & 0.2513 & 0.259 & 0.2652 & 0.2745 & 0.2789 & 0.2899 \\
\hline 0.4 & 0.2681 & 0.2733 & 0.2874 & 0.2939 & 0.3066 & 0.3146 & 0.3256 & 0.3352 & 0.3444 & 0.3558 \\
\hline 0.5 & 0.3146 & 0.3189 & 0.3329 & 0.3458 & 0.3633 & 0.3716 & 0.3874 & 0.3974 & 0.4113 & 0.4262 \\
\hline 0.6 & 0.3627 & 0.3683 & 0.3922 & 0.3993 & 0.4216 & 0.4303 & 0.4508 & 0.4613 & 0.4799 & 0.4922 \\
\hline 0.7 & 0.4125 & 0.4185 & 0.4472 & 0.4547 & 0.4817 & 0.4908 & 0.5159 & 0.5269 & 0.5502 & 0.5631 \\
\hline 0.8 & 0.4643 & 0.4707 & 0.5041 & 0.5119 & 0.5437 & 0.5533 & 0.5832 & 0.5946 & 0.6224 & 0.6359 \\
\hline 0.9 & 0.5184 & 0.5251 & 0.5632 & 0.5716 & 0.6079 & 0.6181 & 0.6525 & 0.6645 & 0.6968 & 0.7109 \\
\hline 1.0 & 0.5748 & 0.5819 & 0.6247 & 0.6336 & 0.6745 & 0.6853 & 0.7242 & 0.7369 & 0.7736 & 0.7885 \\
\hline
\end{tabular}

\section{Conclusion}

The equation (41) represents solution of equation (22) together with condition (23) and (24) by using Homotopy analysis method. Which gives saturation of injected water in inclined porous matrix during instability phenomenon occurred in secondary oil recovery process which satisfy both condition (23) and (24). The solution is in form of exponential function $(\mathrm{X})$ and function of time $\mathrm{T}$ for given perturb parameter $\varepsilon \in[0,1]$. Hence solution shows that saturation of injected water increases exponentially as distance $\mathrm{X}$ (length of average cross-sectional area of schematic fingers) increases for given $\mathrm{T}>0$. From given finger 5 we can concluded that the saturation of injected water is not steadily increasing for small distance $X$ due to the angle of inclination and gravitational effect but after some small distance the saturation of water is steadily exponentially increases as distance $\mathrm{X}$ increases for given time $\mathrm{T}>0$. When it is flowing through interconnected capillaries and external injecting force.

In deduction, for horizontal porous matrix for inclination angle $\theta=0^{\circ}$ gives governing equation (42) together with same condition (23) and (24), the solution (43) represents the saturation of injected water in horizontal porous matrix with same guess value of injected water saturation. The graph of solution (43) given by Figure 6 is exponentially increases from common interface $\mathrm{X}=0$ because here gravitational force and angle of inclination does not play any role.

The comparative study of saturation of injected water for inclined and without incline porous matrix have been given by Figure 5 and 6 . Which shows that saturation of injected water is increasing as distance $\mathrm{X}$ increase for given time $\mathrm{T}>0$. But due inclination the saturation of injecting water is slightly less than the saturation of injected water for case of without inclination for some distance $\mathrm{X}$ increases for given time $\mathrm{T}>0$.

Hence overall we can conclude that when water is injected in oil formatted area at common interface then instability phenomenon occurred and saturation of injected water is increase as distance $\mathrm{X}$ increase for given time $\mathrm{T}>$ 0 in case of incline porous matrix and horizontal porous matrix. But due to inclination the saturation of injected water is slightly less comparative to without inclination. Which is quite obvious for physical as well as experimental results.

\section{Reference}

[1] Bear, J., Dynamics of Fluids in Porous Media, American Elsevier Publishing Co., New York, pp. 687-701, 1972.

[2] Booth, R.J., Miscible flow through porous media .PhD thesis, University of Oxford page-5-10, 2008.

[3] Chuoke, R.L., van Meurs, P. and van der Poel, C., The Instability of Slow, Immiscible Viscous Liquid-Liquid Displacements in Permeable Media, Trans., AIME 216, 188-94, 1959.

[4] Dake. L. P., The Practice of Reservoir Engineering, Elsevier Science B. V., Amsterdam, 1994.

[5] Darcy H., Les Fontaines publiques de lo ville de Dijon, Dalmont, Paris, 1856.

[6] Joshi M.S. and Mehta M.N., Solution by Group Invariant method of Instability phenomenon arising in fluid flow through porous media. International Journal of Engineering Research and Industrial Applications, Vol. 2, No. 1, 35-48, 2009.

[7] Kinjal R. P., Mehta M. N. and Patel T. R., An approximate solution of Instability phenomenon in heterogeneous porous media with mean pressure., Gen. Math. Notes., Vol. 10 (2), 9-21, 2012.

[8] Liao S. J., A kind of approximate solution technique which does not depend upon small parameters (II): an application in fluid mechanics, Int. J. Nonlinear. Mech, 32-815, 1997.

[9] Liao, S. J., An explicit, totally analytic approximation of Blasius viscous flow problems. Intl J. Non-Linear Mech. 34, 759-778, 1999 a.

[10] Liao S. J., On the Homotopy analysis method for nonlinear problems. Appl. Math. Comput. Vol. 147, pp. 499-513, 2004.

[11] Liao, S. J., The proposed homotopy analysis technique for the solution of nonlinear problems. PhD thesis, Shanghai Jiao Tong University, 1992.

[12] Meher R. K. and Mehta M. N. Meher S. K., Instability phenomenon arising in double phase flow through porous media with capillary pressure., Int. J. of Appl. Math and Mech. 7 (15): 97-112, 2011. 
[13] Mehta M. N., Asymptotic expansion of fluid flow through porous media, Ph.D. thesis South Gujarat University, Surat, India, 1977.

[14] Muskat M., Physical principles of oil production, Mc-graw-hill Book co., Inc New York, 1949.

[15] Oroveanu T., Scurgerea Fluiidelor Prin Medii Poroase Neomogene, Editura Academiei Republicii Populare Romine 92, 328, 1963.

[16] Patel T. R. and Mehta M. N., A classical solution of the Burger's equation arising into the instability phenomenon in double phase flow through homogenous porous media, International Journal of Applied Mathematics, Bulgaria, Vol. 25 (1), 2008.

[17] Saffman, P.G. and Taylor, G., The Penetration of a Fluid into a Porous Medium or Hele-Shaw Cell Containing a more Viscous Liquid, Proc. Roy. Soc. London A 245, 312-329, 1958.

[18] Scheidegger A. E., The physics of flow through Porous Media, University of Toronto Press, 1960.
[19] Scheidegger A. E. and Johnson E. F., The statistical behaviour of instabilities in displacement process in porous media., Canadian $J$. Physics, 39-326, 1961.

[20] Vyas N. B., Patel K. R., Mehta M. N. and Patel T. R., Power series solution of instability phenomena in double phase flow through porous media under magnetic field effect, Int. J. of Appl. Math and Mech. 7 (16): 1-10, 2011.

[21] Verma A. P., Statistical behavior of fingering in a displacement process in heterogeneous porous medium with capillary pressure. Can. J. Phys., 47 (3), 319-324, 1969. 